\title{
Management of antifungal susceptibility testing in Italy: comparative results of 2 nationwide surveys (1999 and 2004) in 102 Italian hospitals
}

\author{
Claudio Farina $^{\mathrm{a}, \mathrm{h}, *}$, Esther Manso ${ }^{\mathrm{b}, \mathrm{h}}$, Silvana Sanna ${ }^{\mathrm{c}, \mathrm{h}}$, Gianluigi Lombardi ${ }^{\mathrm{d}, \mathrm{h}}$, \\ Elisabetta Faggie, ${ }^{\text {eh }}$, Paolo Fazii ${ }^{\text {f,h }}$, Stefano Andreoni ${ }^{\text {g,h }}$ \\ aUO Microbiologia, AO "Ospedale San Carlo Borromeo", 20153 Milano, Italia \\ ${ }^{\mathrm{b}}$ Laboratorio Analisi Chimico-Cliniche e Microbiologia, A.O.-U "Ospedali Riuniti di Ancona”, 60100 Ancona, Italia \\ ${ }^{\mathrm{c}}$ Dipartimento di Scienze Biomediche, Sezione di Microbiologia Sperimentale e, Clinica, Università degli Studi, 07100 Sassari, Italia \\ ${ }^{\mathrm{d}}$ Laboratorio di Microbiologia, "Ospedale di Circolo e Fondazione Macchi”, Università degli Studi dell'Insubria, 21100 Varese, Italia \\ e Dipartimento di Sanità Pubblica, Sezione Microbiologia, Università degli Studi di Firenze, 50100 Firenze, Italia \\ ${ }^{\mathrm{f}}$ Laboratorio Analisi Chimico-Cliniche e Microbiologia, P.O. "Spirito Santo", 65100 Pescara, Italia \\ ${ }^{\mathrm{g}}$ Laboratorio di Microbiologia e Virologia, A.O. "Maggiore della Carità", 28100 Novara, Italia \\ ${ }^{\mathrm{h}}$ Medical Mycology Committee, Associazione Microbiologi Clinici Italiani \\ Received 7 April 2006; accepted 22 June 2006
}

\begin{abstract}
The purpose of this study was to verify the standard procedures and minimum level of knowledge of Italian public laboratories involved in the management of antifungal susceptibility testing (AST). Two nationwide surveys were performed in 1999 and 2004 . One hundred and two Italian hospitals located in 85 provincial capitals (82.5\%) participated to these surveys. In 1999, 28 (27.5\%) laboratories versus 16 $(15.7 \%)$ in 2004 stated that they did not perform any susceptibility testing. Some discrepancies observed in the survey confirm that AST is difficult to be correctly managed, and that it can be performed only in very well-trained centers. The great variability of the results of MIC determination and clinical interpretation underlines the urgent need to improve knowledge about indications, method choice, and interpretative criteria for AST both for clinical microbiologists and clinicians.
\end{abstract}

(C) 2007 Elsevier Inc. All rights reserved.

Keywords: Management; Antifungal susceptibility testing; Italy; Surveys; Laboratory

Susceptibility testing for antifungal drugs is a challenge for microbiologists because of the increasing importance of clinicians' requests and the lack of standardization in technology and in interpretative criteria. However, a reference method for antifungal susceptibility testing (AST) of yeasts (Blanc et al., 2005) by the means of a broth dilution technique was approved in 2002 by the Clinical Laboratory Standard Institute (CLSI), formerly known as the National Committee for Clinical Laboratory Standards (NCCLS, 2002), and in 2003 by the European Committee on Antimicrobial Susceptibility Testing (EUCAST, 2003).

\footnotetext{
* Corresponding author. UO Microbiologia, AO "Ospedale San Carlo Borromeo", 20153 Milano, Italia. Tel.: +39-024-022-2456; fax: +39-024022-2829.

E-mail address: farina.claudio@sancarlo.mi.it (C. Farina).
}

The Medical Mycology Committee of the Association of Italian Clinical Microbiologists proposed a national survey to verify the procedures used in the clinical laboratories of public hospitals in Italy. In 1999 and in 2004, 180 questionnaires were mailed to the directors of microbiology laboratories of public hospitals in 103 cities all over Italy. Only public hospital laboratories in province capitals were selected as representative of the average mycological knowledge of both clinicians and microbiologists.

At the end of both studies, the same number of questionnaires $(102 / 180,56.7 \%)$ were returned from $85 / 103(82.5 \%)$ province capitals, even if the geographic location was different between the surveys. In 1999, $28(27.5 \%)$ laboratories versus $16(15.7 \%)$ in 2004 stated that they did not perform any susceptibility testing (Jones et al., 2005). On the contrary, $17(16.7 \%)$ in 1999 versus $13(12.7 \%)$ in 2004 confirmed that AST was part of the routine work. In 57 (55.9\%) versus $73(71.6 \%)$ hospitals 
Table 1

Microbiologic criteria adopted to perform AST (2004 survey)

\begin{tabular}{lccc}
\hline Specimen & $\begin{array}{l}\text { Routinely } \\
\text { done }\end{array}$ & $\begin{array}{l}\text { Never } \\
\text { done }\end{array}$ & $\begin{array}{l}\text { Upon clinician's } \\
\text { request }\end{array}$ \\
\hline Blood & 74 & 4 & 8 \\
CSF & 71 & 7 & 8 \\
Feces & 3 & 68 & 15 \\
Urine & 3 & 26 & 57 \\
Pharyngeal swab & 10 & 54 & 22 \\
Nasal swab & 8 & 57 & 21 \\
Sputum & 18 & 42 & 26 \\
Sterile fluids & 32 & 28 & 26 \\
Vaginal exudate & 11 & 39 & 17 \\
Urethral exudate & 14 & 54 & 18 \\
Skin and annexes & 14 & 55 & 17
\end{tabular}

$\mathrm{CSF}=$ cerebrospinal fluid.

(in 1999 and 2004, respectively), AST was performed only in selected clinical cases.

AST was performed on the basis either of clinician's request in $27 / 57(47.4 \%)$ versus $29 / 73$ (39.7\%) or of microbiologist's decision in the remaining cases (in 1999 and 2004, respectively). Table 1 reports when and which kind of clinical specimen is considered suitable for AST in case of a positive culture.

Comparing results of 1999 versus 2004, disk diffusion was used in $6(8.1 \%)$ versus $1(1.2 \%)$ laboratory; manual systems for MIC detection were performed by $58(78.4 \%)$ versus $82(95.3 \%)$ laboratories. Ten $(13.5 \%)$ versus $3(3.6 \%)$ laboratories declared to use both systems. The adopted commercial methods are reported in Table 2. MICs were evaluated according to the CLSI M27-A2 document. Eleven $(10.8 \%)$ versus $3(3.6 \%)$ laboratories adopted different diagnostic techniques in association.

Tested drugs are generally representative of the used commercial kits.

Sixty of seventy-four (81.1\%) versus 70/86 (81.4\%) of the laboratories declared to report to clinicians all the tested drugs (in 1999 and in 2004, respectively), whereas 5 (6.7\%) versus $6(7.0 \%)$ considered the AST results an exclusive laboratory testing not to be transmitted to clinicians. However, 9 (12.2\%) versus 10 (13.7\%) (in 1999 and in 2004 , respectively) laboratories confirmed to report to clinicians only the results of selected drugs such as amphotericin B, flucytosine, fluconazole, itraconazole, and ketoconazole.

The recent development of new antimycotic drugs improved the rates of recovery from systemic mycoses. ASTs are more frequently requested by clinicians; however, they still represent a true challenge for microbiologists, particularly because of the lack of standardization in interpretative criteria.

In a similar survey performed in 1992 on 94 laboratories in 73 province capitals, $18.1 \%$ of participants performed AST on all isolates, whereas $54.3 \%$ only in some cases (Farina et al., 1995).
Disk diffusion technique was the most commonly used method (30.9\%) in 1992, although occasionally, the laboratory did not follow standard procedures. This was especially true for the choice of medium, because very often, only either Sabouraud dextrose agar or blood agar was used. Commercial kits were very often used, whereas automated systems were not so common (7.4\%).

Most laboratories $(76.1 \%)$ reported to clinicians all the tested antifungal drugs, regardless of their clinical use (systemic or local), of the isolated fungus, and of the clinical specimen; $8.5 \%$ of laboratories did not report any result, and the remaining $15.4 \%$ only reported some antifungal drugs (mainly flucytosine) and only in specific cases.

The survey shows that the same percentage $(27.5 \%)$ of participants in both 1992 and 1999 declare that they do not perform AST, whereas this number decreased to $15.7 \%$ in 2004. The percentage of laboratories that perform AST on all isolates $(16.7 \%)$ or only in some cases $(55.9 \%)$ were similar in 1992 and 1999. The analysis of the 2004 survey shows that the ratio of laboratories performing AST changed, probably because of a better microbiologist education. In fact, the percentage of laboratories that never perform AST had decreased to $12.7 \%$, whereas $71.6 \%$ run AST in some selected cases.

As in the past, in vitro tested drugs are those included in the commercial panels. Data about the adopted criteria for reporting of results are very similar (in both 1999 and 2004) to those observed in 1992, showing that sometimes the microbiologists do not give the clinicians an appropriate answer based on the clinical use of the drug (either systemic or local), the isolated fungus, and the clinical specimen type.

If the interpretative criteria were often "homemade" (criteria suggested by manufacturers rather than the CLSI recommendations: $45.9 \%$ versus $54.1 \%$, in 1992 and 1999 , respectively), the last survey shows that only $5.8 \%$ of laboratories do not follow CLSI (2002) (NCCLS, 2002) or EUCAST (2003) recommendations.

Even if no fundamental changes occurred in indications and interpretation of AST from the early 1990s, many technical improvements have been observed. Compared with the 1992 survey, more attention was found in 1999 in the choice of medium, but actually, disk diffusion is only

Table 2

Manual testing (2004)

\begin{tabular}{lc}
\hline ATB Fungus & $12(15.2 \%)$ \\
\hline Etest & $8(10.1 \%)$ \\
Candifast & $5(6.3 \%)$ \\
Sensititre & $38(48.1 \%)$ \\
Etest + Sensititre & $5(6.3 \%)$ \\
ATB Fungus + Sensititre & $5(6.3 \%)$ \\
Fungitest & $4(5.1 \%)$ \\
ATB Plus & $1(1.3 \%)$ \\
ATB Fungus +Etest & $1(1.3 \%)$ \\
Total & 79
\end{tabular}


sporadically used. Commercial kits were very frequently used: the 2004 data confirm the 1999 results $(95.3 \%$ versus $78.4 \%$, respectively), showing the particular trend of the Italian market. Few laboratories $(10 / 74,13.5 \%)$ used more than 1 technique.

The situation in Italy is quite similar to the one in the United States: Sensititre and Etest together dominate the market $(64.5 \%)$, and a similar distribution between the 2 techniques is observed in both Countries. In fact, Sensititre is the most commonly used technique in both Italy $(60.7 \%)$ and the United States (59\%), followed by Etest $(18.7 \%$ versus 15\%) (Espinel-Ingroff et al., 1998). ATB Fungus is only used by $15.2 \%$ of the Italian laboratories, and only $1.2 \%$ perform disk diffusion methods. On the contrary, in France, the most frequently used methods are ATB Fungus and Etest ( $56 \%$ and $41 \%$, respectively), or a combination of 2 or more methods $(38 \%)$, and $10 \%$ of laboratories perform agar diffusion testing (Blanc et al., 2005).

In conclusion, it seems that Italy largely prefers new technologies, confirming the interest in the progresses in the field and the presence of an important commercial market. However, AST is difficult to be correctly managed, and it could be performed in particular clinical cases only in very well-trained centers, even if standardization is now largely better than in the past (Negroni et al., 2000; Reilly et al., 1999). Moreover, the low level of criticism in reporting the results suggests that mycology have to be taught both as a microbiologic and technical discipline and as a part of infectious disease programs, to improve knowledge about indications, method choice, and interpretative criteria for AST both for clinical microbiologists and clinicians. This concern is confirmed by several nationwide surveys in Italy (Farina et al., 2000) and in France (Eloy et al., 2005).

\section{References}

Blanc V, Eloy O, Sanchez R, Mallié M (2005) Antifungal susceptibility testing in 62 French hospitals. J Mycol Méd 15:197-210.

Eloy O, Blanc V, Mallié M, Decousse JW, Pina P, Allouch PY (2005) Identification and antifungal susceptibility testing of two strains of Candida in 95 French hospitals. J Mycol Méd 15:117-126.

Espinel-Ingroff A, Barchiesi F, Hazen KC, et al (1998) Standardization of antifungal susceptibility testing and clinical relevance. Med Mycol 36(S1):68-78.

European Committee on Antimicrobial Susceptibility Testing (2003) Method for the determination of minimum inhibitory concentration (MIC) by broth dilution of fermentative yeasts. Clin Microbiol Infect 9:1-8.

Farina C, Picerno G, Medical Mycology Committee of AMCLI (1995) Status of medical mycology in clinical laboratories in Italy. Alpe Adria Microbiol J 3:197-209.

Farina C, Mazzoni A, Andreoni S, Faggi E, Fazii P, Lombardi G, Manso E (2000) Gestione dei test di chemiosensibilitià agli antifungini in Italia. Microbiol Med 15:420-425.

Jones RN, Fritsche TR, CAP-MRC Members (2005) Accuracy for antibacterial and antifungal susceptibility tests: report from the College of American Pathologists (CAP) Microbiology Proficiency Survey Program for 2004-2005. 45th Interscience Conference on Antimicrobial Agents and Chemotherapy, Washington, DC, December 16-19, poster D-1654/75.

National Committee for Clinical Laboratory Standards (NCCLS) (2002) Reference method for broth dilution antifungal susceptibility testing of yeasts. Approved Standard M27-A2, (2nd ed.)Wayne (PA): National Committee for Clinical Laboratory Standards.

Negroni R, Ellis D, Bulmer G, et al (1998) Teaching medical mycology in the year 2000. Med Mycol 36(S1):106-108.

Reilly AA, Salkin IF, McGinnis MR, et al (1999) Evaluation of mycology laboratories proficiency testing. J Clin Microbiol 37:2297-2305. 